\title{
Are histomorphologic changes in the fimbrial ends more to blame for primary epithelial ovarian carcinomas than initially thought?
}

\author{
Gokce ASKAN ${ }^{1}$ (D) Ipek ERBARUT SEVEN ${ }^{2}$ (D), Naziye OZKAN ${ }^{2}$ (D), Funda EREN ${ }^{2}$ (D) \\ ${ }^{1}$ Department of Pathology, Rize University, Training and Research Hospital, Rize, Turkey. \\ ${ }^{2}$ Department of Pathology, School of Medicine, Marmara University, Istanbul Turkey.
}

Corresponding Author: Gokce ASKAN

E-mail: gokceaskan@gmail.com

Submitted: 24.08.2021 Accepted: 21.10.2021

\begin{abstract}
Objective: To investigate the relationship between primary epithelial ovarian tumors and histomorphologic changes in the fimbrial ends (FEs) of the fallopian tubes.

Materials and Methods: Twenty-eight serous carcinomas (SCs) and 12 non-serous carcinomas (NSC) were studied. Ovarian and concomitant invasive tumors in FEs were labeled with PAX8, WT-1 and Calretinin.

Results: Eighty-six percent of SCs were high grade (HG), 14\% of were low grade (LG). 71\% of SCs (85\% HG, 15\% LG) had concomitant invasive tumors in FEs. Serous tubal intraepithelial carcinoma (STIC) was seen in 29\% (75\% HG, 25\% LG), all had concomitant invasive tumors in FEs. The presence of tumors in FEs was statistically significant in SCs ( $\mathrm{p}=0.03$ ). $33 \%$ of NSCs had concomitantly invasive tumors in FEs. $67 \%$ of endometrioid tumors, 33\% of clear cell carcinomas had endometriosis. $50 \%$ of mucinous tumors, $67 \%$ of endometrioid tumors, 50\% of benign Brenner tumors had Walthard nest. Except for mucinous carcinomas, ovarian and concomitant invasive tumors in FEs displayed tubal phenotype (Calretinin-/PAX8+).

Conclusion: The results of our study suggest that, invasive tumors and STIC in FEs are not only limited to HGSCs, but can also be seen in LGs. FEs could also be a site of origin for NSCs, however, future studies with more cases are needed.

Keywords: Primary epithelial ovarian tumors, Fallopian tubes, Fimbrial ends, Histomorphologic changes, Early detection
\end{abstract}

\section{INTRODUCTION}

Epithelial ovarian cancer constitutes the second most common gynecological malignancy with highest mortality in women [1, 2]. The high mortality rate is primarily due to the difficulties in diagnosis, including the lack of specific and sensitive biomarkers that could aid early diagnosis. Studies have shown that epithelial ovarian cancer is not a single disease, but it is composed of a diverse group of tumors that can be classified based on different morphologic and molecular features [3-5]. One group of tumors, designated type 1 , is composed of low-grade serous carcinomas (LGSCs), low-grade endometrioid, clear cell, mucinous and transitional cell carcinomas [6,7]. Another group of tumors, designated type 2 , is highly agressive and includes conventional high-grade serous carcinomas (HGSCs), undifferentiated carcinomas and malignant mixed mesodermal tumors [3]. The most common subtype of ovarian carcinoma is serous carcinomas, and HGSCs comprise the vast majority. Thus, most of the studies have focused on HGSCs, and LGSCs have not been well-studied. LGSC is a unique entity with clinical and molecular characteristics distinct from HGSCs. Compared to HGSCs, LGSCs tend to occur in younger women, have indolent behavior and are chemoresistant [8]. In the last decade, studies have shown that HGSCs are likely derived from the epithelium of fimbrial ends (FEs). In contrast to HGSCs, it was assumed that, LGSCs arose from ovarian epithelial inclusions, serous cystadenoma or adenofibroma, and then progressed to serous borderline tumors and invasive carcinoma [9-13]. However, a few recent studies explored some histomorphological changes in the FEs of fallopian tubes, and concluded that secretory cells in fallopian tubes could be the source of LGSCs [14-16].

The aim of our study is to determine histomorphological findings (metaplasia, epithelial atypia, serous tubal intraepithelial

How to cite this article: Askan G, Erbarut Seven I, Ozkan N, Eren F. Are histomorphologic changes in the fimbrial ends more to blame for primary epithelial ovarian carcinomas than initially thought? Marmara Med J 2022; 35(1):23-30. doi: 10.5472/marumj.1056169 
carcinoma (STIC), invasive tumor), and evaluate any relationship between histologic types of ovarian carcinomas and these changes by using Senctioning and Extensively Examining the Fimbriated End (SEE-FIM) protocol for sampling fallopian tubes in groups with tumours and in control groups.

\section{MATERIALS and METHODS}

Our project was reviewed in 2012, and ethical approval was obtained from the Marmara University, School of Medicine Clinical Research Ethics Committee (approval number: 09.20120170).

Forty primary epithelial ovarian carcinomas between January 2011 and May 2014 were retrieved from the files. Cases with tumors that directly invaded the FEs were excluded from the study. Forty cases with tissue diagnosis of leiomyoma within the same period were included as the control group.

Serous carcinomas were graded according to M.D. Anderson criteria [17]. Tumor cells consist of uniform, round to oval nucleus with distinct nucleoli, mild to moderate nuclear atypia, and the mitotic index of up to 12 mitoses per 10 high power fields were classified as low-grade, while tumor cells with distinct pleomorphism, severe nuclear atypia, nucleus with coarsed chromatin pattern and mitotic index of more than 12 mitoses per high power were filed as high-grade [17]. Then, according to the p53 staining pattern, cases were reclassified as low or high grade.

Fimbrial ends of 80 cases (40 from tumor group and 40 from control group) were firstly evaluated by hematoxylin and eosin stain, to see if there were any histomorphological findings which replaced the normal tubal epithelium. Suspicious foci were reevaluated with serial tissue sections. Stratification with epithelial atypia, loss of cilia, hyperchromasia, presence of nucleolus, increased nucleocytoplasmic ratio and loss of polarity were considered significant for STIC.

Sectioning and Extensively Examining the Fimbriated End protocol was used for sampling fallopian tubes of all cases including the control group [18-20]. The best representative formalin-fixed paraffin-embedded tissue section (for tumor group; ovarian tumor and concomitant invasive tumor in FEs, for control group; normal appearing FEs) was chosen in each case. Three micrometer thickness sections were obtained and underwent overnight deparaffinization at $37^{\circ} \mathrm{C}$. The sections were submerged for antigen retrieval in citrate buffer (ph 6.0). Immunohistochemical staining was performed with streptavidine biotin peroxidase method by using antibodies as follows: Calretinin (1:50, 5A5, Novocastra, Leica Biosystems, UK), PAX8 (1:200, MRQ50, Cell Marque, California, US), WT-1 (1:200, 6F-H2, Cell Marque, California, US) and p53 (1:800, DO7; Novocastra, Leica Biosystems, UK). Nuclear labeling in at least $25 \%$ of cells was considered to be expression for PAX8 and WT1 , and cytoplasmic for Calretinin. p53 was applied to FEs of both the tumor group and the control group. Regardless of presence of atypia, diffuse linear nuclear staining of equal to or more than 12 epithelial cells was considered significant for p53 signature.

\section{Statistical Analysis}

Fisher's exact test was used for statistical analysis, and $\mathrm{p}$ values of $<0.05$ were considered statistically significant.

\section{RESULTS}

Distrubution of tumor types was summarized in Table I. Twenty eight (70\%) cases were serous carcinomas (SCs), and 12 (30\%) were non-serous carcinomas (NSCs). Median age in the tumor and control group was 56.2 years and 50.4 years respectively.

\section{Serous carcinomas}

Twenty four (86\%) were HGSCs and 4 (14\%) were LGSCs. Median ages of HGSCs and LGSCs 59 years and 56.5 years respectively. Concomitant invasive tumors were seen in FEs of 20 SCs (71\%); 17 (85\%) were HGSCs and 3 (15\%) were LGSCs (Figure 1). STIC was seen in eight (29\%) cases; six were (75\%) HGSCs, two (25\%) were LGSCs (Figure 2A), and all had concomitant invasive tumors in FEs (Figure 2B). There was no statistically significant difference between tumor grade and STIC or presence of invasive tumors in FEs $(p=0.29$ and $p=0.68)$. However, the presence of concomitant invasive tumors in FEs was statistically significant in SCs, compared to NSCs ( $\mathrm{p}=0.03$ ) (Table II).

Table I. Distribution of tumor types

\begin{tabular}{|lc|}
\hline Histologic tumor type & $\begin{array}{c}\text { Number of cases } \\
\mathbf{n}(\%)\end{array}$ \\
\hline Serous carcinoma & $28(70)$ \\
Mucinous tumor & $4(10)$ \\
Clear cell carcinoma & $3(7.5)$ \\
Endometrioid tumor & $3(7.5)$ \\
Brenner tumor & $2(5)$ \\
Total number of cases & 40 \\
\hline
\end{tabular}

Table II. Comparison of the presence of tumors at the fimbrial ends between serous and non-serous carcinomas

\begin{tabular}{|lc|}
\hline Histologic tumor type (n) & $\begin{array}{c}\text { The presence of tumor at the fimbrial end } \\
\mathbf{n}(\%)\end{array}$ \\
\hline Serous carcinoma (28) & $20(71.4)$ \\
High grade serous carcinoma (24) & $17(85)$ \\
Low grade serous carcinoma (4) & $3(15)$ \\
Non-serous carcinoma (12) & $4(33)$ \\
Mucinous tumor (4) & 0 \\
Clear cell carcinoma (3) & $1(25)$ \\
Endometrioid tumor (3) & $2(50)$ \\
Brenner tumor (2) & $1(25)$ \\
\hline p=0.03
\end{tabular}

p53 signature was seen in $11(39 \%)$ cases (Figure 3A); eight (73\%) had concomitant invasive tumor in FEs (Figure 3B), and three (27\%) had STIC (Figure 3C). The presence of p53 signature and tumor grade was not significantly associated $(\mathrm{p}=0.48)$. 
All SCs were positive for PAX-8 (Figure 4A), 24 (86\%) were positive for p53 (Figure 4B), and $24(86 \%)$ were positive for WT-1 (Figure 4C). Calretinin was also positive in five (18\%) cases (Figure $4 \mathrm{D})$, and three $(60 \%)$ of them were high-grade (Table III). The ovarian tumors and invasive tumors in FEs displayed tubal phenotype (Calretinin- $\backslash \mathrm{PAX} 8+$ ) in 18 (90\%) cases.

Table III. Staining pattern of ovarian tumors in all cases

\begin{tabular}{|lcccc|} 
Histologic tumor type (n) & $\begin{array}{c}\text { p53 } \\
\mathbf{n}(\%)\end{array}$ & $\begin{array}{c}\text { PAX8 } \\
\mathbf{n}(\%)\end{array}$ & $\begin{array}{c}\text { WT-1 } \\
\mathbf{n}(\%)\end{array}$ & $\begin{array}{c}\text { Calretinin } \\
\mathbf{n}(\%)\end{array}$ \\
\hline Serous carcinoma (28) & $24(86)$ & $28(100)$ & $24(86)$ & $5(18)$ \\
Mucinous tumor (4) & 0 & 0 & 0 & 0 \\
Clear cell carcinoma (3) & $1(33)$ & $1(33)$ & $1(33)$ & 0 \\
Endometrioid tumor (3) & 0 & $2(67)$ & $2(67)$ & $1(33)$ \\
Brenner tumor (2) & $1(50)$ & 0 & $1(50)$ & 0 \\
\hline
\end{tabular}

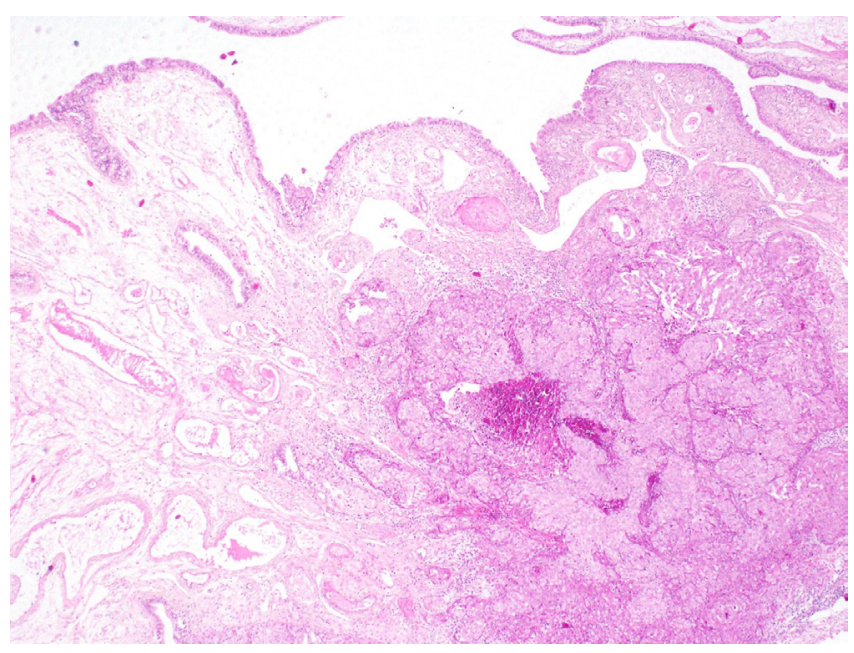

Figure 1. Presence of tumor in the fimbrial end in serous carcinoma (H\&E, X40)

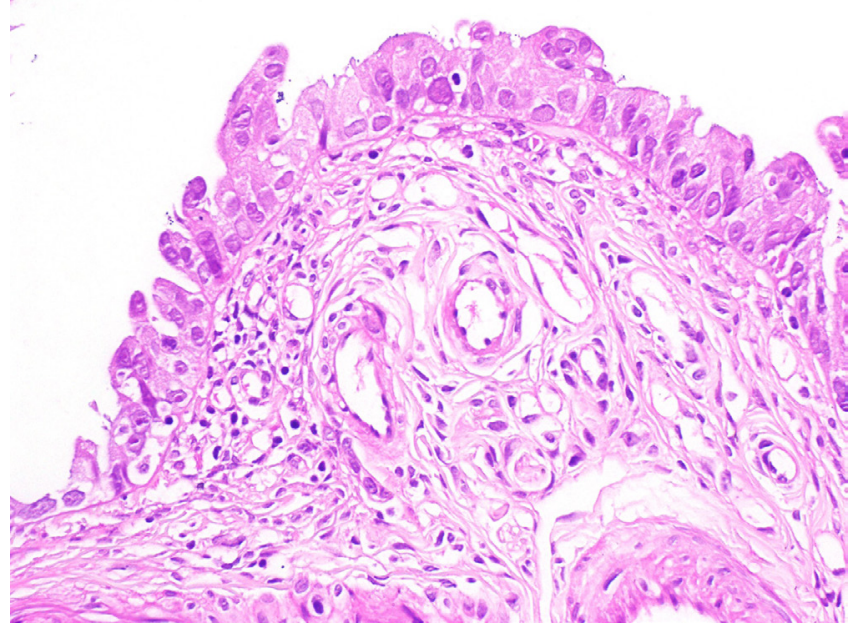

Figure 2A. Presence of STIC in serous carcinomas (H\&E, X200)

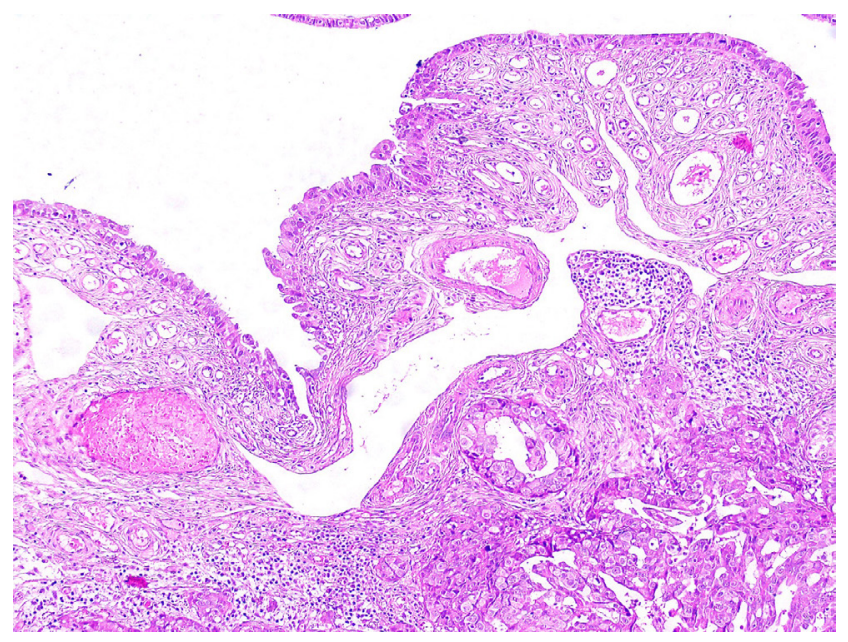

Figure 2B. Presence of STIC and concomitant invasive tumor in the fimbrial end (H\&E, X100)
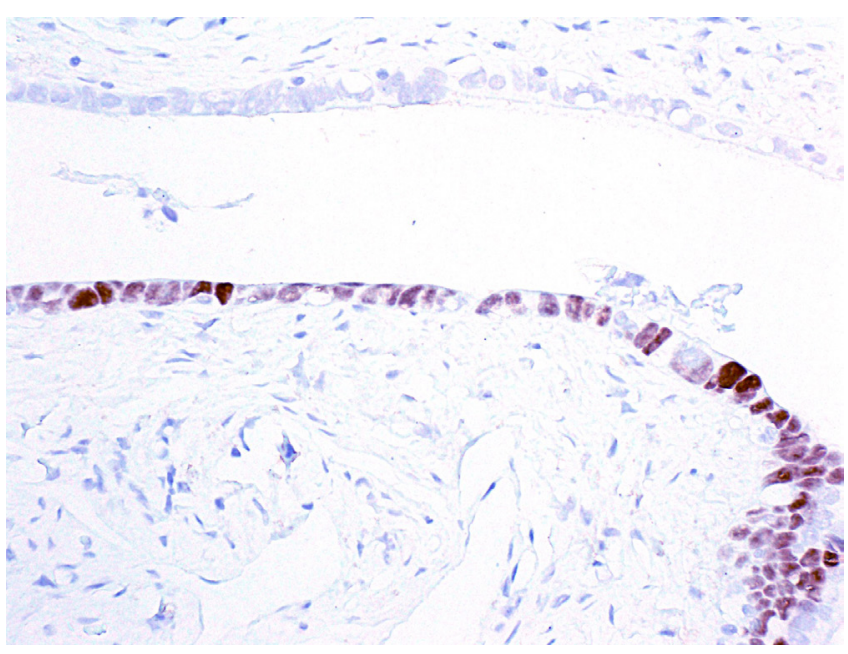

Figure $3 A$. p53 signature (X200)

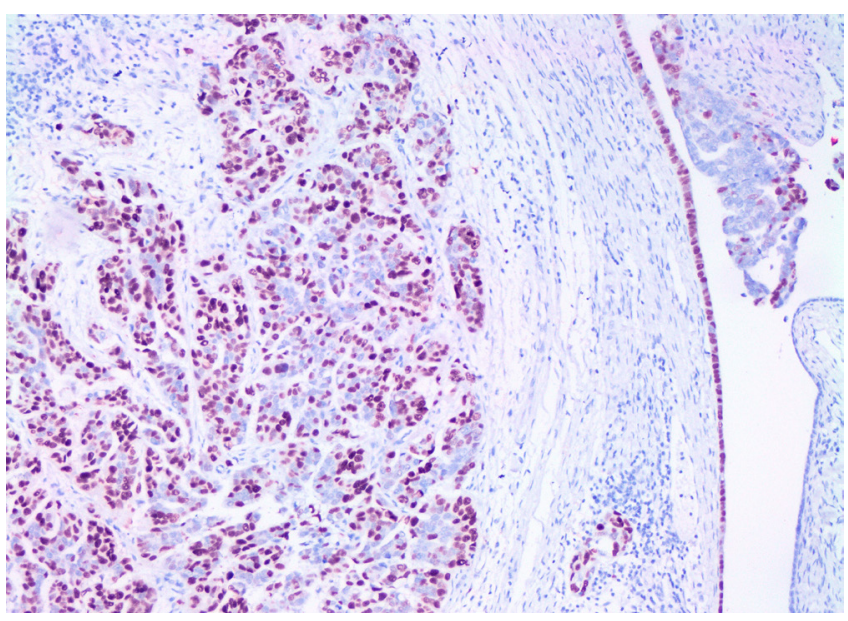

Figure 3B. p53 signature and concomitant invasive tumor in the fimbrial end $(X 100)$ 


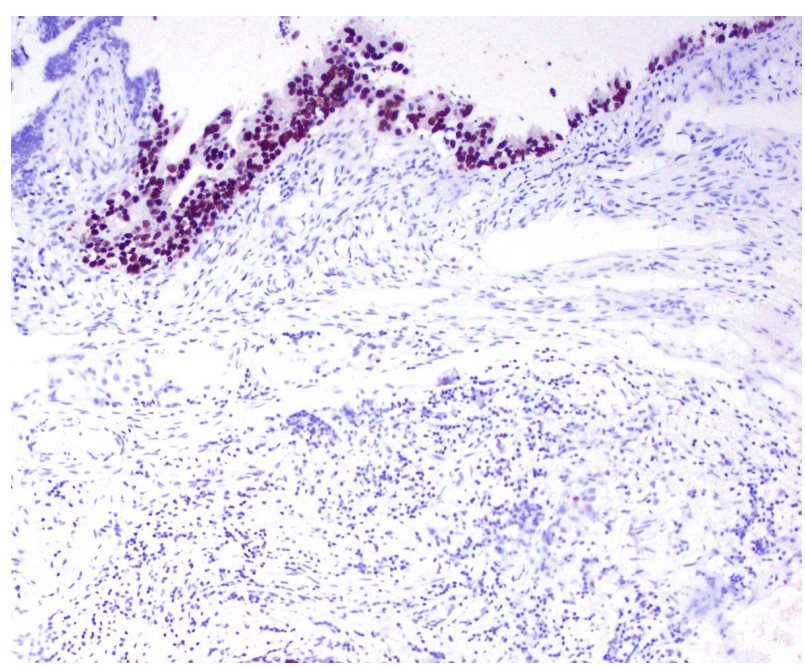

Figure 3C. Presence of STIC and p53 expression (X100)

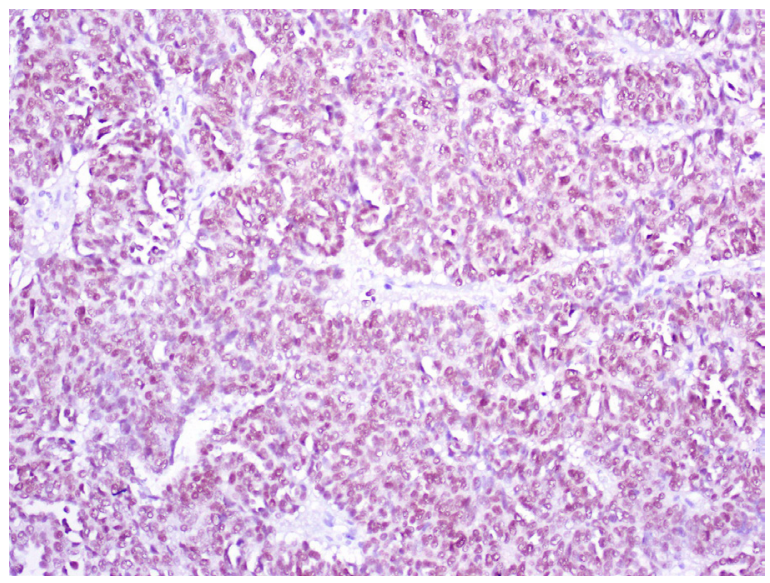

Figure 4A. Expression of serous carcinomas for PAX-8 (X100

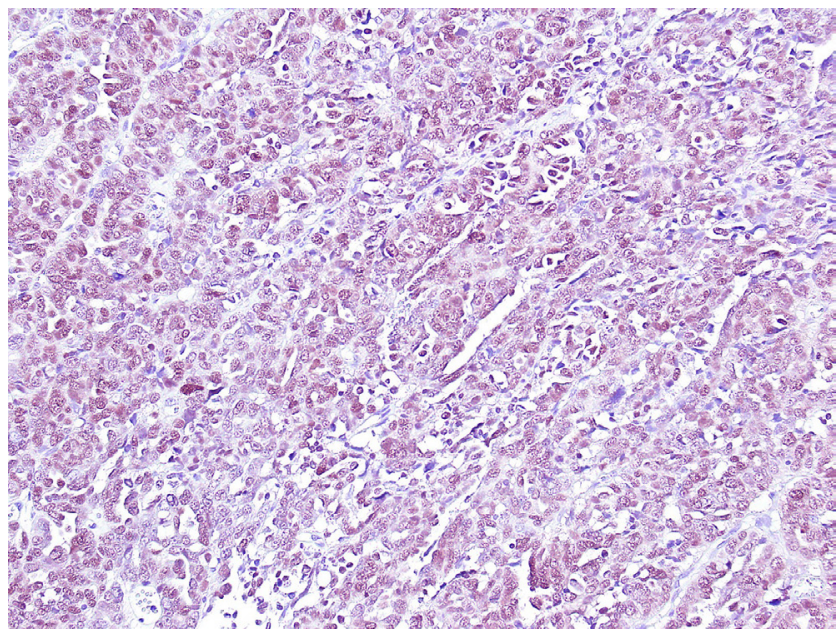

Figure 4B. Expression of serous carcinomas for p53 (X100)

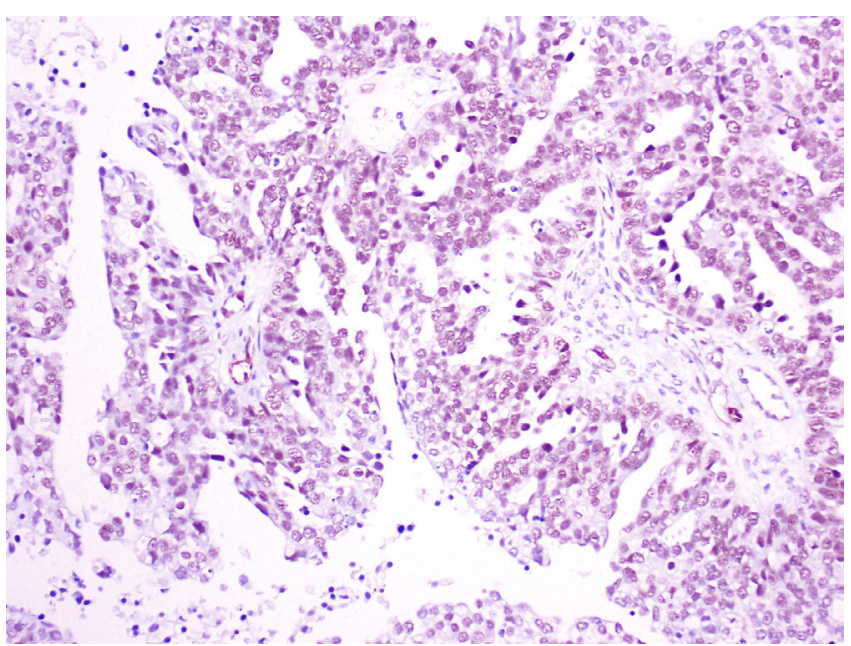

Figure 4C. Expression of serous carcinomas for WT-1 (X200)

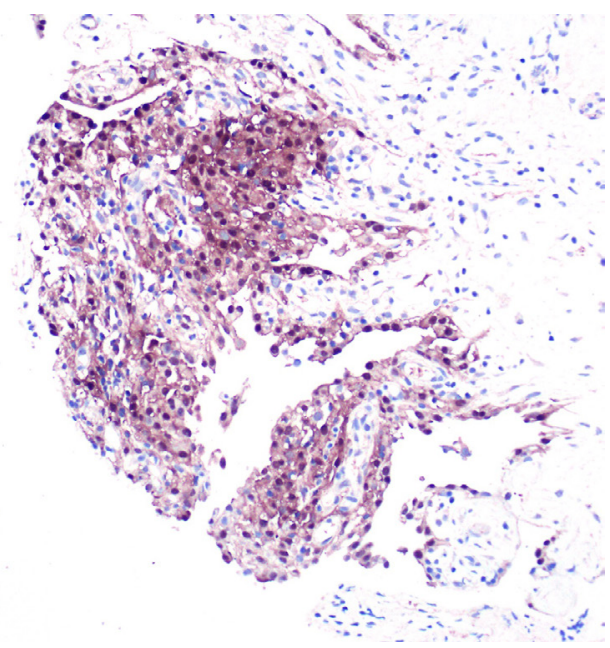

Figure 4D. Expression of serous carcinomas for Calretinin (X200)

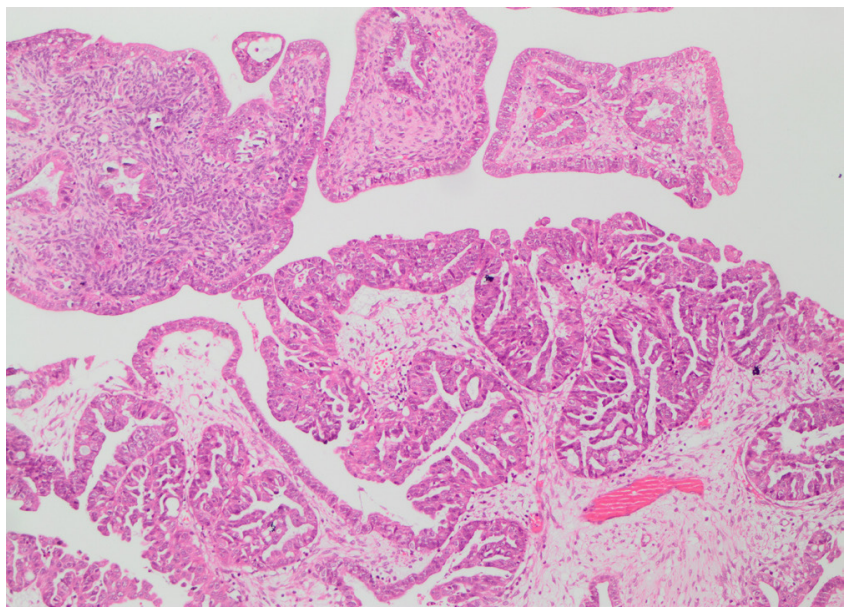

Figure 5A. Endometrioid tumor (H\&E, X100) 


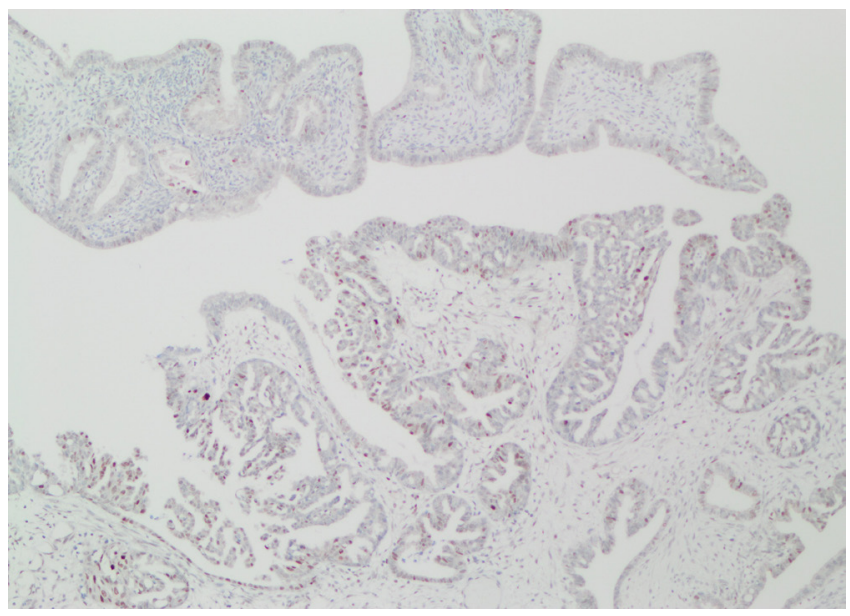

Figure 5B. Expression of endometrioid tumor for p53 (H\&E, X100)

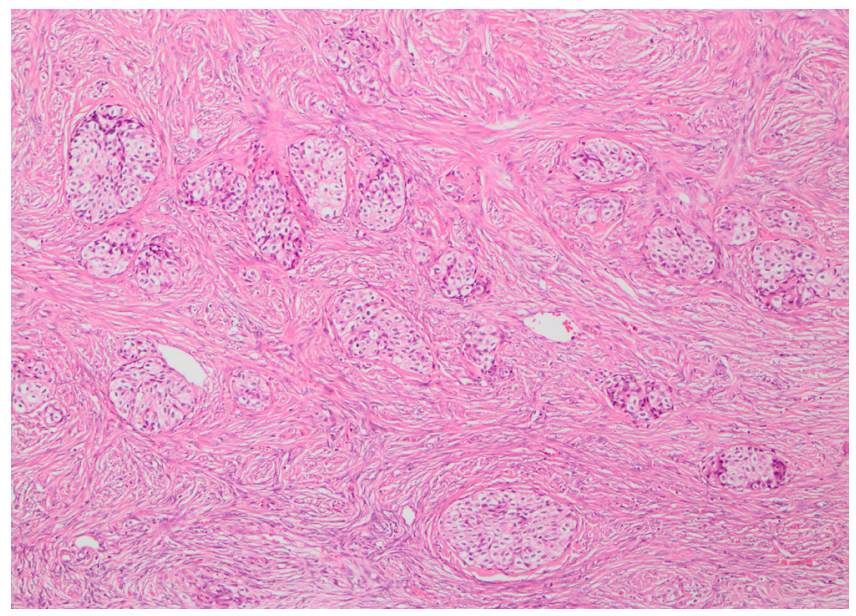

Figure 6A. Brenner tumor (H\&E, X100)

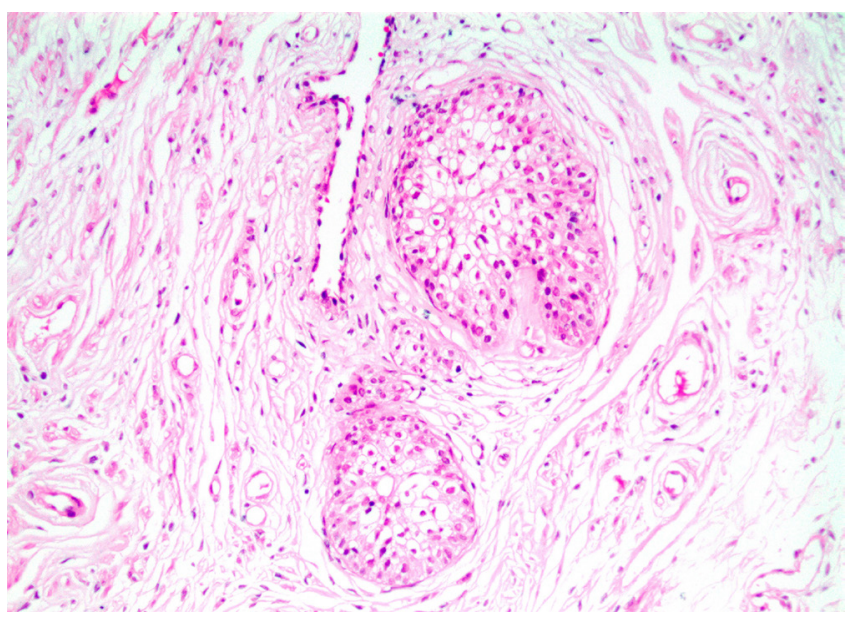

Figure 6B. Brenner tumor and concomitant invasive tumor in the fimbrial end (HઐE, X200)

\section{Non-serous carcinomas}

A total of 12 cases were NSCs including four (33\%) mucinous tumors, three (25\%) clear cell carcinomas, three (25\%) endometrioid tumors and two (17\%) benign Brenner tumors. Three (75\%) mucinous tumors and two (67\%) endometrioid tumors were borderline. The concomitant invasive tumors in FEs were seen in four (33\%) cases; two endometrioid tumors (Figure 5A and 5B), one clear cell carcinoma and one benign Brenner tumor (Figure 6A). No invasive tumor or TIC was seen in the FEs of mucinous tumors. Although, this ratio is less than in SCs, more studies with a larger number of cases should be carried out for equitable comparison. One (8\%) case had TIC, and five (42\%) had p53 signature. There was no statistically significant difference in presence of $\mathrm{p} 53$ signature between SCs and NSCs $(\mathrm{p}=0.58)$ (Table IV). Endometrioid tumors $(67 \%)$ which had invasive tumors in FEs, had also endometriosis foci in FEs. No in situ carcinoma was seen in FEs and one had p53 signature. The clear cell carcinoma case which had invasive tumor in FEs, had also TIC and endometriosis foci. The benign Brenner tumor case which had invasive tumors in FEs (Figure 6B) had also p53 signature and Walthard nest in FEs. Walthard nest was also seen in $50 \%$ of mucinous tumors and $67 \%$ of endometrioid tumors. Except mucinous tumors, ovarian tumors and concomitant invasive tumors in the FEs displayed tubal phenotype (Calretinin-\PAX8+) in all cases.

Table IV. Comparison of p53 signature between serous carcinomas and non-serous carcinomas

\begin{tabular}{|lc|}
\hline Group $(\mathbf{n})$ & p53 signature $\mathbf{n}(\%)$ \\
\hline Serous carcinomas (28) & $11(39)$ \\
Non-serous tumors (12) & $5(42)$ \\
Total number of case (40) & 16 \\
\hline
\end{tabular}

$p=0.58$

Table V. Comparison of the presence of tumors at the fimbrial end in the literature and in our study

The name of the study
Tumor at the fimbrial end (\%)

Mehra et al 2011 [19] 80

Medeiros et al 2006 [18]

Salvador et al 2009 [20]

Our study

\section{DISCUSSION}

The current study is one of the few studies investigating the fallopian tubal epithelial pathologies in SCs graded by M.D Anderson criteria. The incidence of invasive tumor in the FEs of HGSCs (85\%) is significantly higher than in LGSC (15\%). As a result, we found that $\mathrm{FE}$ could be the origin of not only the HGSCs but also the LGSCs.

Women with BRCA germ line mutations, who had undergone prophylactic salphingooophorectomy, were studied for targeted region of serous carcinogenesis in previous studies (Table V). 
While Mehra et al., found invasive tumors in FEs in $80 \%$ of the cases, Medeiros et al., identified invasive tumours in $38 \%$ of women with BRCA germ line mutations [20,21]. Salvador et al., studied sporadic SCs. They found invasive tumors in FEs in $58 \%$ of cases, and all were HGSCs [23]. These studies have provided evidence that the fallopian tube, especially the fimbria is the primary site of most HGSC. In our study, we found a high percentage $(71 \%)$ of invasive tumors in FEs of SCs, and $85 \%$ of them were HG.

The origin of LGSC is less clear compared to HGSCs, as there were only few studies related to this tumor group $[8,12$, $14,15]$. The few LGSC can be the main issue for this. Also, different grading systems among these studies prevent accurate comparison.

The theory that SCs originate from fallopian tubal epithelia was further supported by the presence of a high percentage (59\%) of STIC accompanied with invasive tumors in HGSCs. However, LGSCs were not mentioned in these studies [24, 25]. In our study, the incidence of STIC was $21 \%$, and invasive tumors in FEs of HGSCs and LGSCs were $85 \%$ and $15 \%$ respectively. [9, 21]. This showed that invasive tumors and STIC could be found in LGSCs as well as in HGSCs.

In literature, the association between p53 signature and STIC was reported in a wide range (20-60\%) [26]. In our study, p53 signature was seen in 11 (39\%) SCs; three (27\%) had STIC, and eight $(73 \%)$ had concomitant invasive tumor in FEs. This difference can be explained by different sampling methods of FEs, as in most of the studies no information was given about the sampling method. Presence of STIC or invasive tumor in FEs among all cases, where p53 signature was identified, gave rise to the opinion that HGSCs are originated from this sequence $[9,11]$. We found that p53 signature could be identified independently from tumor grade and was not only specific for HGSCs, but also can be seen in LGSCs and NSCs.

The precursors of STIC, identified as p53 signature, was reported approximately $10-40 \%$ within normal population in literature. Most studies reported that the ratio of this incidence can be changed by studying with different ethnic groups and sampling methods. By sampling with SEE-FIM protocol, the incidence was found higher $[19,24]$, and in our study p53 signature was identified in six (15\%) of control group which was compatible with literature.

In additon to histomorphologic features, performing immunohistochemical stains including PAX8, WT-1 and Calretinin, was suggested to determine the origin of tumor. It is thought that the tumor is originated from fallopian tubal epithelium, if it is positive for PAX8 and negative for Calretinin, while it is originated from mesothelium if stained vice versa [15, $25,26]$. In our study, ovarian tumor and concomitant invasive tumor in FEs showed tubal phenotype in 18 (90\%) SCs. Five (18\%) SCs were positive for Calretinin. The significant relationship with HGSCs and Calretinin positivity were reported in recent studies, and this was explained by loss of tumor differentiation. In our study, $60 \%$ of Calretinin positive carcinomas were high grade. In addition, it has also been proposed that, some ovarian tumors arise from the mesothelial surface lining of the ovaries or from invaginations of this lining into the superficial ovarian cortex that form cortical inclusion cysts. Thus, these inclusion cysts were blamed for precursor lesions of ovarian carcinomas [30]. However, to make an exact comment, we thought that histomorphological changes and staining pattern of ovarian inclusion cysts should also be studied.

A few studies about NSCs located in FE were reported. However, the fimbriae were not examined in detail in any of these cases. Alvarado-Cabrero et al., studied SCs and NSCs located in FE, but in this study, the survey was evaluated, instead of the histomorphological findings of the fimbriae [28]. Maeda et al., studied 52 primary ovarian carcinomas, and invasive tumors in FEs were only seen in SCs [29]. Seidmann et al., studied Brenner tumors, and no invasive tumor or precursor lesion was found in the FEs. However, Walthard nests were evident in $20 \%$ of cases. By detailed sampling of the FEs and examining the precursor lesions, comments about the origin of mucinous and Brenner tumors could also be made [30]. According to the results of these studies, it was thought that NSCs could also have originated from the FEs $[31,32]$. In our study, concomitant invasive tumors in the FEs were seen in four (33\%) NSCs; two (\%50) were endometrioid tumors, one (\%25) was clear cell carcinoma, and one (\%25) was benign Brenner tumor. No concomitant invasive tumor in the FEs was seen in the mucinous tumors. Mucinous tumors were also different from other types of tumors by their staining pattern, since they displayed mesothelial phenotype (Calretinin+\PAX8-). The limitation of our study was too few cases for studying the relationship between NSCs and FEs. However, we thought that detailed sampling of FEs in all ovarian carcinomas was important for obtaining precursor lesions. Except for mucinous tumors, the relationship between FE and NSCs may be found by studying more cases.

\section{Conclusion}

Our findings suggest that most SCs, regardless of tumor grade, originate from FEs. Although, the number of cases is quite low, our study suggests that NSCs can also originate from FEs. To determine the relationship between NSCs and FEs, more cases should be studied with detailed sampling method. The results of all these studies are helpful for both classification of primary ovarian epithelial carcinomas and precursor lesions. Also, recognising the origin of precursor lesions could light the way for ideas on more conservative surgeries, which is vital for reproductive women.

\section{Compliance with the Ethical Standards}

Ethical Approval: The study protocol was approved by the Marmara University, Faculty of Medicine, Ethics Committee with the (approval number: 09.20120170).

The study was conducted in accordance with the principles of the Declaration of Helsinki.

Financial Support: The authors have no relevant financial information to close. 
Conflict of Interest: The authors have no potential conflicts of interest to disclose.

Author contributions: GA and FE: Conception and design of the study, drafting the article, GA, FE, IE and NO: Data collection and writing, GA and FE :Analysis and interpretation of data, FE and IES: Revising the article critically for intellectual content. All authors read and approved the final version of the article.

Author contributions: GA and FE: Conception and design of the study, drafting the article, GA, FE, IE and NO: Data collection and writing, GA and FE :Analysis and interpretation of data, FE and IES: Revising the article critically for intellectual content. . All authors read and approved the final version of the article.

\section{REFERENCES}

[1] Kurman R. In: Hedrick Ellenson L, Ronnett, Brigitte M, eds. Blaustein`s Pathology of The Female Genital Tract: Springer, 2019.

[2] Kurman RJ. Origin and molecular pathogenesis of ovarian high-grade serous carcinoma. Ann Oncol 2013;24 (Suppl 10):x16-21. doi:10.1093/annonc/mdt463.

[3] Nik NN, Vang R, Shih Ie M, Kurman RJ. Origin and pathogenesis of pelvic (ovarian, tubal, and primary peritoneal) serous carcinoma. Annu Rev Pathol 2014;9:27-45. doi:10.1146/ annurev-pathol-020.712.163949.

[4] McCluggage WG. Morphological subtypes of ovarian carcinoma: a review with emphasis on new developments and pathogenesis. Pathology 2011;43:420-32. doi: 10.1097/ PAT.0b013e328348a6e7.

[5] Kurman RJ. In: WHO Classification of Tumours Editorial Board eds. WHO Classification of Tumours of the Female Genital Tumors, 5th ed, Vol. 4: World Health Organization, 2020.

[6] Conic I, Dimov I, Tasic-Dimov D, Djordjevic B, and Stefanovic V. Ovarian epithelial cancer stem cells. Scientific World Journal 2011;11:1243-69. doi: 10.1100/tsw.2011.112.

[7] Reichman ME, Altekruse S, Li CI, et al. Feasibility study for collection of HER2 data by National Cancer Institute (NCI) Surveillance, Epidemiology, and End Results (SEER) Program central cancer registries. Cancer Epidemiol Biomarkers Prev 2010;19:144-47. doi: 10.1158/1055-9965.EPI-09-0807.

[8] Gershenson DM. Low-grade serous carcinoma of the ovary or peritoneum. Ann Oncol 2016;27(Suppl 1):i45-i49. doi:10.1093/annonc/mdw085.

[9] Vang R, Shih Ie M, and Kurman RJ. Fallopian tube precursors of ovarian low - and high-grade serous neoplasms. Histopathology 2013;62:44-58. doi:10.1111/his.12046.

[10] Crum CP. Intercepting pelvic cancer in the distal fallopian tube: theories and realities. Mol Oncol 2009;3:165-70. doi:10.1016/j.molonc.2009.01.004.

[11] Carlson JW, Miron A, Jarboe EA, et al. Serous tubal intraepithelial carcinoma: its potential role in primary peritoneal serous carcinoma and serous cancer prevention. J Clin Oncol 2008;26:4160-65. doi:10.1200/JCO.2008.16.4814.

[12] Rabban JT, Krasik E, Chen LM, Powell CB, Crawford B, and Zaloudek CJ. Multistep level sections to detect occult fallopian tube carcinoma in risk-reducing salpingo-oophorectomies from women with BRCA mutations: implications for defining an optimal specimen dissection protocol. Am J Surg Pathol 2009;33:1878-85. doi:10.1097/PAS.0b013e3181bc6059.

[13] Kurman RJ and Shih Ie M. The origin and pathogenesis of epithelial ovarian cancer: a proposed unifying theory. Am J Surg Pathol 2010;34:433-43. doi: 10.1097/PAS.0b013e3181cf3d79.

[14] Laury AR, Ning G, Quick CM, et al. Fallopian tube correlates of ovarian serous borderline tumors. Am J Surg Pathol 2011;35:1759-65. doi: 10.1097/PAS.0b013e318233b0f7.

[15] Li J, Abushahin N, Pang S, et al. Tubal origin of 'ovarian' lowgrade serous carcinoma. Mod Pathol 2011;24:1488-99. doi: 10.1038/modpathol.2011.106.

[16] Wang Y, Hong S, Mu J, et al. Tubal Origin of "Ovarian” LowGrade Serous Carcinoma: A Gene Expression Profile Study. J Oncol 2019:2019:1-9. doi: 10.1155/2019/8659754.

[17] Malpica A, Deavers MT, Lu K, et al. Grading ovarian serous carcinoma using a two-tier system. Am J Surg Pathol 2004;28:496-504. doi: 10.1097/00000.478.200404000-00009.

[18] Arora S, Yelikar BR, Karigoudar MH. Evaluation of SEE-FIM (Sectioning and Extensively Examining the Fimbriated End) protocol in identifying fallopian tube precursor lesions in women with ovarian tumors. J Obstet Gynaecol 2019;69:15359. doi: 10.1007/s13224.018.1155-z.

[19] Nomura H, Ikki A, Fusegi A, et al. Clinical and pathological outcomes of risk-reducing salpingo-oophorectomy for Japanese women with hereditary breast and ovarian cancer. Int J Clin Oncol 2021. doi: 10.1007/s10147.021.02020-9.

[20] Cheng A, Li L, Wu M, et al., Pathological findings following risk-reducing salpingo-oophorectomy in BRCA mutation carriers: A systematic review and meta-analysis. Eur J Surg Oncol 2020;46:139-47. doi: 10.1016/j.ejso.2019.09.002.

[21] Medeiros F, Muto MG, Lee Y, et al. The tubal fimbria is a preferred site for early adenocarcinoma in women with familial ovarian cancer syndrome. Am J Surg Pathol 2006;30:230-36. doi: 10.1097/01.pas.000.018.0854.28831.77.

[22] Mehra KK, Chang MC, Folkins AK, et al. The impact of tissue block sampling on the detection of p53 signatures in fallopian tubes from women with BRCA 1 or 2 mutations (BRCA+) and controls. Mod Pathol 2011;24:152-56. doi: 10.1038/ modpathol.2010.171

[23] Salvador S, Gilks B, Kobel M, Huntsman D, Rosen B, and Miller D. The fallopian tube: primary site of most pelvic highgrade serous carcinomas. Int J Gynecol Cancer 2009;19:58-64. doi: 10.1111/IGC.0b013e318199009c.

[24] Lynch HT, Casey MJ, Snyder CL, et al. Hereditary ovarian carcinoma: heterogeneity, molecular genetics, pathology, and management. Mol Oncol 2009;3:97-137. doi:10.1016/j. molonc.2009.02.004 
[25] Kurman RJ, McConnell TG. Precursors of endometrial and ovarian carcinoma. Virchows Arch 2010;456:1-12. doi:10.1007/s00428.009.0824-9.

[26] Crum CP, Drapkin R, Miron A, et al. The distal fallopian tube: a new model for pelvic serous carcinogenesis. Curr Opin Obstet Gynecol 2007;19:3-9. doi: 10.1097/GCO.0b013e328011a21f.

[27] Walsh T, Casadei S, Lee MK, et al. Mutations in 12 genes for inherited ovarian, fallopian tube, and peritoneal carcinoma identified by massively parallel sequencing. Proc Natl Acad Sci U S A 2011;108:18032-37. doi: 10.1073/ pnas.111.505.2108.

[28] Soslow R. In: Goldblum J eds. Current Concepts in Gynecologic Pathology: Epithelial Tumors of the Gynecologic Tract, An Issue of Surgical Pathology Clinics. 1st ed,. Vol. 4; Saunders, 2011.

[29] Cuatrecasas M, Catasus L, Palacios J, Prat J. Transitional cell tumors of the ovary: a comparative clinicopathologic, immunohistochemical, and molecular genetic analysis of Brenner tumors and transitional cell carcinomas. Am J Surg Pathol 2009;33:556-67. doi: 10.1097/PAS.0b013e318188b84c.

[30] Adler E, Mhawech-Fauceglia P, Gayther SA, and Lawrenson K. PAX8 expression in ovarian surface epithelial cells. Hum Pathol 2015;46:948-56. doi: 10.1016/j.humpath.2015.03.017.
[31] Alvarado-Cabrero I, Navani SS, Young RH, Scully RE. Tumors of the fimbriated end of the fallopian tube: a clinicopathologic analysis of 20 cases, including nine carcinomas. Int J Gynecol Pathol 1997;16:189-96. doi: 10.1097/00004.347.19970700000001.

[32] Maeda D, Ota S, Takazawa Y, et al. Mucosal carcinoma of the fallopian tube coexists with ovarian cancer of serous subtype only: a study of Japanese cases. Virchows Arch 2010;457:597-608. doi: 10.1007/s00428.010.0979-4. Epub 2010 Sep 25.

[33] Seidman JD, Zhao P, Yemelyanova A."Primary peritoneal" high-grade serous carcinoma is very likely metastatic from serous tubal intraepithelial carcinoma: assessing the new paradigm of ovarian and pelvic serous carcinogenesis and its implications for screening for ovarian cancer. Gynecol Oncol 2011;120:470-73. doi: 10.1016/j.ygyno.2010.11.020.

[34] Liu F, Wei J, Shen D, Liu J. Mucinous borderline tumor involving fallopian tube: case report and review of the literature. Int J Clin Exp Pathol 2013;6:962-65.

[35] Kobayashi H, Kajiwara H, Kanayama S, et al. Molecular pathogenesis of endometriosis-associated clear cell carcinoma of the ovary (review). Oncol Rep 2009;22: 233-40. doi: 10.3892/ or 00000429 . 